\title{
ESTIMATIVA DE RISCO DE CONTAMINAÇÃo DAS ÁGUAS POR PESTICIDAS NA REGIÃO SUL DO ESTADO DO RS
}

\author{
Liziara Cabrera*, Fabiane Pinho Costa e Ednei Gilberto Primel \\ Escola de Química e Alimentos, Universidade Federal do Rio Grande, CP 475, 96201-900 Rio Grande - RS, Brasil
}

Recebido em 17/9/07; aceito em 20/6/08; publicado na web em 5/11/08

\begin{abstract}
RISK ESTIMATE OF WATER CONTAMINATION BY PESTICIDE IN SOUTH OF BRAZIL. Pesticides can be quite useful in agricultural production, however, some pesticides after application can reach and contaminate the hydric resource. Pesticide monitoring must be careful because it is expensive and lengthy. Thus, in this work, a theoretical prediction was made using the approaches suggested by EPA-USA, the Groundwater Ubiquity Score index (GUS) and the Goss method associated with physicochemical properties of 27 pesticides used in a region in the south of Brazil. The results of this work led to the identification of the pesticides that should be prioritized in the environmental monitoring due to their high potential for contaminating water resources.
\end{abstract}

Keywords: theoretical prediction; pesticides; water contamination.

\section{INTRODUÇÃO}

A produção agrícola, principalmente em escala comercial, sempre esteve associada ao uso de pesticidas com a finalidade de evitar e combater pragas que geram perdas na colheita. Os principais produtos usados comercialmente são compostos orgânicos sintéticos com alta atividade biológica, entre estes, destacam-se, os inseticidas, fungicidas e herbicidas que em geral são tóxicos, podendo ser cancerígenos e causadores de mutações. ${ }^{1}$

Com o crescimento demográfico, a demanda de alimentos aumentou, portanto, para garantir alimentos suficientes e de qualidade à população, faz-se o uso de pesticidas..$^{2,3}$ Esses compostos são aplicados em quantidades significativas e em áreas extensas tendo como principal destino o meio ambiente. ${ }^{3-5}$

A contaminação por pesticidas ocorre tanto pontualmente como nas áreas adjacentes, podendo atingir até mesmo locais mais distantes do ponto de aplicação. Dependendo de suas características os pesticidas podem permanecer em diferentes compartimentos ambientais, tais como atmosfera, solo, água de superfície e subterrânea. Através das suas propriedades físico-químicas, pode-se estimar seu comportamento no meio ambiente, desde a aplicação até o destino final, assim como as interações com o solo e o transporte, quando dissolvidos em água ou associados ao sedimento. ${ }^{1,6,7}$ Alguns pesticidas podem ser degradados por vias químicas, fotólise ou ação de microrganismos. Entretanto, as moléculas com alta persistência (baixa taxa de degradação) podem permanecer no ambiente sem sofrer qualquer alteração. ${ }^{3}$

Os recursos hídricos são os mais afetados devido à agricultura exigir um suprimento de água, o que conduz o desenvolvimento dessa atividade próximo a rios e lagos. ${ }^{8-10} \mathrm{O}$ comprometimento destes recursos naturais gera graves prejuízos à saúde humana, principalmente quando estes são utilizados como fonte para abastecimento público. ${ }^{10-12}$ Tal situação exige controle e estudos que possibilitem o monitoramento de pesticidas no meio ambiente.

No Brasil, a demanda por pesticidas vem se acentuando a cada ano, destacando-se como um dos maiores consumidores mundiais de pesticidas. ${ }^{13} \mathrm{O}$ estado do Rio Grande do Sul (RS) em 2006 foi responsável por $10,4 \%$ do consumo nacional de pesticidas, ${ }^{14}$ devido

\footnotetext{
*e-mail: liziara.cabrera@ vetorial.net
}

à intensa atividade agrícola, com destaque para o cultivo de arroz irrigado. ${ }^{15}$ Dentre os 1,3 milhões de hectare de arroz irrigado cultivados no Brasil, cerca de $73 \%$ encontram-se no RS, contribuindo com cerca de $50 \%$ da produção nacional de arroz. Essa atividade demanda um elevado consumo de água, equivalente a 80\% da água consumida no estado. ${ }^{16}$

No extremo sul do RS, nas cidades de Rio Grande (RG) e de Santa Vitória do Palmar (SVP) uma das atividades predominantes é o cultivo de arroz irrigado, com destaque para o município de SVP que produz cerca de 500 mil t/ano, sendo juntamente com o município de Uruguaiana os maiores produtores nacionais de arroz, o município de RG destaca-se também com uma produção anual de 85 mil t. ${ }^{15}$

Neste estudo foram realizadas entrevistas com comerciantes agrícolas e com produtores rurais, com objetivo de traçar um diagnóstico dos principais pesticidas utilizados na região. Este diagnóstico resultou em uma listagem de 27 compostos em uso nas atividades agrícolas nas cidades de RG e SVP (Tabela $1 \mathrm{~S}$, material suplementar). Para estes pesticidas foram aplicados critérios para avaliar a possibilidade de contaminação dos recursos hídricos, utilizando suas propriedades físico-químicas. Para avaliar o potencial de contaminação, de águas superficiais e subterrâneas, utilizaram-se três critérios: O critério de Screening da Agência de Proteção Ambiental dos Estados Unidos (US-EPA), a medida do índice de vulnerabilidade de águas subterrâneas (GUS) e a aplicação do Método de Goss. Tais análises possibilitam indicar quais compostos apresentam risco de contaminar os recursos hídricos contribuindo, assim, na elaboração de um planejamento para estudos de monitoramentos de pesticidas em água na região minimizando custo e tempo para o seu desenvolvimento.

\section{PARTE EXPERIMENTAL}

\section{Área de estudo}

O município de Santa Vitória do Palmar situa-se na região extremo sul do RS, fronteira com o Uruguai, com população estimada em 33 mil habitantes, tendo como principal atividade econômica a agropecuária, com destaque para o cultivo de arroz. A orizicultura no município é praticada às margens da porção brasileira da Lagoa Mirim, a qual $97 \%$ da demanda hídrica é para irrigação das lavouras de arroz. ${ }^{17}$ 
O município de Rio Grande, com cerca de 200 mil habitantes situa-se as margens do estuário da Lagoa dos Patos no litoral sul do RS. O cultivo de arroz é também a cultura local predominante, juntamente com cebola e tomate. As lavouras de arroz são localizadas principalmente às margens do canal São Gonçalo, o qual liga a Lagoa Mirim ao estuário da Lagoa dos Patos. Já as demais culturas são predominantes nas adjacências da Ilha dos Marinheiros, Vila da Quinta e no município vizinho de São José do Norte. A Lagoa Mirim liga-se ao estuário da Lagoa dos Patos através do canal São Gonçalo de onde é captada água para o abastecimento da maior parte do município de Rio Grande. ${ }^{17}$ Já na área rural e no Município de Santa Vitória o abastecimento de água é realizado através de poços artesianos. Portanto, estudos que contemplem a conservação tanto de águas de superfície como de água subterrânea são essenciais na região.

A temperatura média na região varia de $12-26^{\circ} \mathrm{C}$, com taxa de precipitação mensal de $70-120 \mathrm{~mm}$ primavera/verão e outono/inverno, respectivamente, a insolação é de cerca de 5 h diárias no outono/ inverno e cerca de $8 \mathrm{~h}$ diárias na primavera/verão. ${ }^{18}$

O solo predominante na região é o neossolo litorâneo eutrófico, vertissolo e chernossolo, sendo este último ideal para plantação de arroz, e apresenta razoáveis teores de matéria orgânica, portanto, neste tipo de solo o pesticida tende a ficar aderido. Há ainda na região ocorrência de solo argiloso, principalmente ao redor de Rio Grande e de planossolo e organossolo ao redor de Santa Vitória do Palmar. Os solos argilosos são menos porosos e, portanto, também tendem a adsorver os contaminantes, já o planossolo apresenta texturas mais arenosas, onde a porosidade é maior, sendo assim os pesticidas são facilmente lixiviados neste tipo de solo. ${ }^{19}$

\section{Caracterização e propriedades físico-químicas}

Além da estrutura e massa molecular, as propriedades físicoquímicas de cada pesticida possibilitam prever o seu comportamento no meio ambiente. As principais propriedades abordadas neste estudo são solubilidade em água, coeficiente de adsorção normalizado pelo carbono orgânico $\left(\mathrm{K}_{\mathrm{oc}}\right)$, produto da constante de dissociação ácida do composto ( $\mathrm{pk}_{\mathrm{a}}$ ), pressão de vapor (PV), constante da lei de Henry $\left(\mathrm{K}_{\mathrm{H}}\right)$, coeficiente de partição octanol-água $\left(\mathrm{K}_{\mathrm{ow}}\right)$, tempo de meia vida $\left(\mathrm{t}_{1 / 2}\right)$ no solo e na água.

A estrutura molecular definida de cada pesticida determina, pelo menos parcialmente, o grau com que ele irá interagir no ambiente. A presença de grupos funcionais contendo oxigênio, nitrogênio e enxofre tendem a tornar os compostos orgânicos mais reativos, tanto química quanto biologicamente, mais solúveis em água e menos voláteis quando comparados aos hidrocarbonetos com o mesmo número de carbonos. A presença de halogênios também torna o composto menos solúvel em água, mais volátil e normalmente menos reativo tornando-o mais estável ambientalmente. ${ }^{1}$

A solubilidade em água é uma propriedade importante para os processos ambientais, pois atua no comportamento, transporte e destino desses compostos, indicando a tendência do pesticida em ser carreado superficialmente no solo atingindo águas superficiais. No entanto, este não é o único parâmetro para prever a percolação, devendo ser analisado em conjunto com outras propriedades. ${ }^{1}$

Os valores do produto da constante de dissociação ácida $\left(\mathrm{pK}_{\mathrm{a}}\right)$ têm efeito sobre a solubilidade do pesticida e, através do $\mathrm{pK}_{\mathrm{a}}$ do pesticida e do $\mathrm{pH}$ do solo, é possível prever a forma predominante (ionizável ou molecular) de um pesticida ácido, a mesma relação pode ser obtida usando $\mathrm{pK}_{\mathrm{b}}$ para pesticidas alcalinos. ${ }^{6}$

Com o coeficiente de adsorção $\left(\mathrm{K}_{\mathrm{oc}}\right)$ é possível prever a tendência do pesticida a ficar adsorvido na matéria orgânica no solo. Moléculas altamente solúveis tendem apresentar valores de $\mathrm{K}_{\mathrm{oc}}$ relativamente baixos (menores que $150 \mathrm{~cm}^{3} \mathrm{~g}^{-1}$ ), podendo ser mais rapidamente biodegradados no solo e na água. ${ }^{6}$

A Constante da Lei de Henry $\left(\mathrm{K}_{\mathrm{H}}\right)$, também chamada de coeficiente de partição ambiental ar-água, juntamente com a pressão de vapor (PV) mostra a tendência do pesticida a volatilizar ou permanecer na fase aquosa. Para algumas moléculas, dependendo do valor da pressão de vapor e/ou valor da solubilidade em água, o $\mathrm{K}_{\mathrm{H}}$ dificilmente pode ser medido. ${ }^{1}$

O coeficiente de partição octanol-água $\left(\mathrm{K}_{\mathrm{ow}}\right)$ relaciona as propriedades hidrofílicas e lipofílicas, demonstrando a tendência à bioconcentração destes compostos, sendo um fator importante na avaliação de riscos, pois em conjunto com os dados de degradação, o potencial de acumulação pode ser usado na identificação dos pesticidas que podem ser transportados via cadeia alimentar. ${ }^{6}$

O tempo de meia vida $\left(\mathrm{t}_{1 / 2}\right)$ é um critério usado para determinar os efeitos ambientais relacionados à volatilização, potencial de lixiviação e características de degradação de vários compostos químicos.

As propriedades descritas anteriormente foram utilizadas para determinar os critérios de avaliação para contaminação de águas superficiais (Goss) e subterrâneas (EPA e GUS) descritos a seguir. ${ }^{20}$

\section{Avaliação da contaminação das águas superficiais}

Para avaliar o potencial de contaminação das águas de superfície por pesticidas foi utilizado o critério de Goss, o qual considera a meiavida do composto no solo $\left(\mathrm{t}_{1 / 2 \text { no solo }}\right)$, sua solubilidade em água à $25^{\circ} \mathrm{C}$ e a constante de adsorção à matéria orgânica do solo $\left(\mathrm{K}_{\mathrm{oc}}\right)$.

Segundo este critério os pesticidas são divididos nos seguintes grupos: Alto Potencial de Transporte das Águas Superficiais devido ao transporte associado ao sedimento em suspensão (APTAS); Baixo Potencial de Transporte das Águas Superficiais devido ao transporte associado ao sedimento em suspensão (BPTAS); Alto Potencial de Transporte das Águas superficiais devido a serem Transportados Dissolvidos na Água (APTDA) e Baixo Potencial de Transporte das Águas Superficiais devido a serem Transportados Dissolvidos na Água (BPTDA). Os valores de cada parâmetro para estes grupos são: APTAS - $\mathrm{t}_{1 / 2 \text { no solo }} \geq 40$ dias e $\mathrm{K}_{\mathrm{oc}}=1000 \mathrm{~cm}^{3} \mathrm{~g}^{-1}$, ou $\mathrm{t}_{1 / 2 \text { no solo }} \geq 40$ dias, $\mathrm{K}_{\mathrm{oc}} \geq 500 \mathrm{~cm}^{3} \mathrm{~g}^{-1} \mathrm{e}$ solubilidade em água $\leq 0,5 \mathrm{mg} \mathrm{L}^{-1}$; BPTAS $-\mathrm{t}_{1 / 2}$ no solo $<1$ dia, ou $\mathrm{t}_{1 / 2 \text { no solo }} \leq 40$ dias, $\mathrm{K}_{\mathrm{oc}} \leq 500 \mathrm{~cm}^{3} \mathrm{~g}^{-1}$ e solubilidade $\geq 0,5 \mathrm{mg} \mathrm{L}^{-1}$, ou $\mathrm{t}_{1 / 2 \text { no solo }} \leq 2$ dias e $\mathrm{K}_{\mathrm{oc}} \leq 500 \mathrm{~cm}^{3} \mathrm{~g}^{-1}$ ou $\mathrm{t}_{1 / 2 \text { no solo }} \leq 4$ dias, $\mathrm{K}_{\mathrm{oc}} \leq 900 \mathrm{~cm}^{3} \mathrm{~g}^{-1}$ e solubilidade em água $\geq 0,5 \mathrm{mg} \mathrm{L}^{-1}$ ou $\leq 40$ dias e $\mathrm{K}_{\mathrm{oc}} \leq 900 \mathrm{~cm}^{3} \mathrm{~g}^{-1}$ e solubilidade em água $\geq 2 \mathrm{mg} \mathrm{L}^{-1}$; APTDA - $\mathrm{t}_{1 / 2 \text { no solo }}>35$ dias, $\mathrm{K}_{\mathrm{oc}}<1.000 .000 \mathrm{~cm}^{3} \mathrm{~g}^{-1} \mathrm{e}$ solubilidade $>1 \mathrm{mg}$ $\mathrm{L}^{-1}$, ou $\mathrm{t}_{1 / 2 \text { no solo }}>35$ dias, $\mathrm{K}_{\mathrm{oc}}<700 \mathrm{~cm}^{3} \mathrm{~g}^{-1} \mathrm{e}$ solubilidade em água entre 10 e $100 \mathrm{mg} \mathrm{L}^{-1}$; BPTDA - $\mathrm{K}_{\mathrm{oc}}>1.000 .000 \mathrm{~cm}^{3} \mathrm{~g}^{-1}$, ou t $\mathrm{t}_{1 / 2 \text { no solo }}$ $<1$ dia e $\mathrm{K}_{\mathrm{oc}}<100 \mathrm{~cm}^{3} \mathrm{~g}^{-1}$, ou $\mathrm{t}_{1 / 2 \text { no solo }}<35$ dias e solubilidade em água $<0,5 \mathrm{mg} \mathrm{L}^{-1}$.

Os compostos que não se enquadram em nenhum destes grupos são considerados com potencial médio, ou seja, Médio Potencial de contaminação das Águas Superficiais devido a serem Transportados Dissolvidos na Água (MPTDA) ou associado ao sedimento em suspensão (MPTAS). ${ }^{20}$

\section{Avaliação da contaminação da água subterrânea}

Os critérios utilizados para avaliar o potencial de lixiviação contaminando a água subterrânea foram os sugeridos pela EPA e o Índice de GUS.

Os critérios da EPA envolvem os valores de solubilidade em água $\left(\right.$ a $\left.25^{\circ} \mathrm{C}\right)>30 \mathrm{mg} \mathrm{L}^{-1}$; coeficiente de adsorção à matéria orgânica do solo $\left(\mathrm{K}_{\mathrm{oc}}\right)<300-500 \mathrm{~cm}^{3} \mathrm{~g}^{-1}$; constante da Lei de Henry $\left(\mathrm{K}_{\mathrm{H}}\right)<10^{-2}$ $\mathrm{Pa} \mathrm{m}^{3} \mathrm{~mol}^{-1}$, a especiação (presença de forma aniônica em $\mathrm{pH}$ normal, entre 5 e 8) e o $t_{1 / 2 \text { solo }}>14-21$ dias e na água > 175 dias; condições de campo que favorecem a percolação no solo, como índice de pluviosi- 
dade anual $>250 \mathrm{~mm}$, aqüífero não confinado e solo poroso.

$\mathrm{O}$ índice GUS inclui o $\mathrm{t}_{1 / 2 \text { no solo }}$ do composto no solo e o $\mathrm{K}_{\mathrm{oc}}$. Sendo calculado como: GUS $=\left(\log t_{1 / 2 \text { no solo }}\right) \cdot\left(4-\log \mathrm{K}_{\mathrm{oc}}\right)$. O resultado deste cálculo possibilita classificar os compostos em três categorias, aqueles que não sofrem lixiviação (IL) GUS $<1,8$; compostos que se encontram na faixa de transição (TL) $1,8<$ GUS $<2,8$ e compostos provavelmente lixiviados (PL) GUS $>2,8 .{ }^{18}$

\section{RESULTADOS E DISCUSSÃO}

Segundo o critério de Goss o pesticida cialofope-butílico apresenta BPTAS e BPTDA. Já os pesticidas 2,4-D, carboxina, fipronil, propanil, tiobencarbe e tiram também apresentam BPTAS, mas apresentam MPTDA, indicando que podem ser transportados na forma dissolvida em água (Tabela 1).

Os pesticidas glifosato, molinato, atrazina, carbofurano, linurom, clomazona e metsulfurom-metílico são provavelmente transportados na forma dissolvida em água (APTDA), sendo que atrazina, carbofurano, glifosato, linurom e metsulfurom-metílico apresentam MPTAS, enquanto que os demais possuem BPTAS (Tabela 1). Propinebe e parationa-metílica possuem baixo BPTAS e MPTDA, respectivamente.

Pendimetalina e permetrina apresentam médio potencial de transporte tanto dissolvidos em água quanto associado ao sedimento em suspensão (MPTAS e MPTDA) (Tabela 1).

Lambda-cialotrina (com APTAS e BPTDA) e trifluralina (com APTAS e MPTDA) podem atingir a água de superfície, preferencialmente adsorvidos ao sedimento.

O potencial de risco de contaminação de águas superficiais, segundo o critério de Goss, não foi avaliado para o bisbiribaque sódico, fenoxaprope-p-etílico, pirazossulfurom, sulfosato e para tebuconazol, pois não há dados suficientes para esta avaliação. Ciclossulfamurom e quincloraque também não foram classificados quanto ao potencial de transporte associado ao sedimento, pois, não possuem dados de meia vida no solo, porém apresentam MPTDA.

Alguns desses compostos já foram determinados em águas de superfície em diferentes regiões, ${ }^{21}$ principalmente atrazina. ${ }^{22}$ No entanto, nas águas da região em estudo até o momento são poucos os dados de monitoramento de pesticidas. ${ }^{23}$ No entanto, já foram determinados alguns pesticidas organoclorados em tecidos animais na região extremo sul do RS. ${ }^{24}$ Ocorrências de pesticidas em água foram estudadas em outras regiões do estado, especialmente na região central do RS. ${ }^{25}$

A ocorrência de pesticidas em água subterrânea vem sendo estudada, ${ }^{26}$ o que reforça o risco de contaminação também deste recurso. Segundo os índices aplicados para prever a contaminação das águas subterrâneas os pesticidas atrazina, carbofurano, clomazona, linurom, molinato, metsulfurom-metílico, pendimetalina e tiram apresentam risco de contaminação segundo o método de GUS, sendo classificados por este método como provavelmente lixiviados (PL). Já os pesticidas 2,4-D e parationa metílica apresentam uma tendência à lixiviação (TL) conforme este mesmo método (Tabela 2).

Segundo os critérios da EPA, além dos compostos já citados, também apresentam risco de contaminação para as águas subterrâneas os seguintes pesticidas: bisbiribaque sódico, carboxina, propanil e o tebuconazol. Tiram e pendimetalina não atendem aos parâmetros da EPA mesmo sendo provavelmente lixiviados considerando o índice de GUS. O contrário ocorre com bisbiribaque sódico, carboxina, glifosato e propanil, os quais apresentam baixos valores de GUS (IL) (Tabela 2).

Provavelmente não apresentam risco de contaminação à água subterrânea os inseticidas fipronil, lambda-cialotrina, e permetrina e os herbicidas ciclossulfamurom, cialofope butílico, pirazossulfurom e tiobencarbe (Tabela 2).

Para o herbicida quincloraque nada pode ser afirmado, pois, na literatura consultada, não há dados de $\mathrm{t}_{1 / 2}$ no solo, no entanto, os valores de $\mathrm{K}_{\mathrm{oc}}$ e $\mathrm{K}_{\mathrm{H}}$ atendem a exigência dos critérios da EPA para prever a contaminação da água subterrânea. Já sulfosato e fenoxapropep-etílico não têm dados suficientes para qualquer pressuposição, no entanto, sulfosato é bastante solúvel, e fenoxaprope-p-etílico é estável e solúvel em água, indicando que estes compostos tendem a permanecer neste compartimento (Tabela 2).

Além da problemática com os princípios ativos em questão, muitos desses compostos ainda produzem metabólitos como, por exemplo, 3,4-DCA, metabólito do propanil e de outros pesticidas, MCPA e 8-hidroxibentazone, metabólitos do bentazone. Esses e outros metabólitos podem ser ainda mais persistentes e mais tóxicos que os princípios ativos. ${ }^{27}$

\section{CONCLUSÕES}

Dos 27 pesticidas utilizados na região, pelo menos 19 apresentam risco de contaminação para águas de superfície e subterrâneas.

Os pesticidas atrazina, carbofurano, clomazona, linurom, metsulfurom-metílico, molinato e glifosato necessitam de um estudo para avaliar sua ocorrência tanto na água superficial, como na subterrânea, pois estes compostos têm alto potencial de poluição nestes dois compartimentos. Os resultados mostram que o monitoramento de pesticidas em águas de superfície na região deve enfocar, além dos pesticidas citados anteriormente, também o pesticida lambda-cialotrina, pois tem potencial de contaminação de águas superficiais (PC) segundo os critérios da EPA. Além desses, é indicado avaliar a ocorrência dos pesticidas 2,4-D, carboxina, propanil, tiobencarbe, tiram e parationametilica, por apresentarem, segundo o critério de Goss, potencial médio de contaminação (MPTDA e/ou MPTAS) à água superficial.

Tabela 1. Estimativa de contaminação de águas de superfície usando o critério de Goss

\begin{tabular}{lccccc}
\hline PESTICIDA & Classificação & PESTICIDA & Classificação & \multicolumn{2}{c}{ PESTICIDA } \\
\hline 2,4-D & BPTAS MPTDA & Fipronil & BPTAS MPTDA & Pirazossulfurom-etílico & ND \\
Atrazina & MPTAS APTDA & Glifosato & MPTAS APTDA & Propanil & BPTAS MPTDA \\
Bispiribaque sódico & ND & Lambda-cialotrina & APTAS BPTDA & Propinebe & BPTAS ND \\
Carbofuran & MPTAS APTDA & Linurom & MPTAS APTDA & Quincloraque & ND MPTDA \\
Carboxina & BPTAS MPTDA & Metsulfurom-metílico & MPTAS APTDA & Sulfosato & ND \\
Cialofope-butilico & BPTAS BPTDA & Molinato & APTDA & Tebuconazol & ND \\
Ciclo-sulfamurom & ND MPTDA & Parationa-metílica & BPTAS MPTDA & Tiobencarbe & BPTAS MPTDA \\
Clomazona & BPTAS APTDA & Pendimetalina & MPTAS MPTDA & Tiram & BPTAS MPTDA \\
Fenoxaprope-p-etílico & ND & Permetrina & MPTAS MPTDA & Trifluralina & APTAS MPTDA \\
\hline
\end{tabular}

APTAS: Alto potencial transporte associado ao sedimento; BPTAS: Baixo potencial de transporte associado ao sedimento; APTDA: Alto potencial de transportados dissolvidos na água; BPTDA: Baixo potencial de transportados dissolvidos na água; MPTAS: Médio potencial de transporte associado ao sedimento; MPTDA: Médio potencial de transportados dissolvidos na água e ND: não determinado. 
Tabela 2. Avaliação de risco de contaminação para água subterrânea usando os critérios da EPA e o índice de GUS

\begin{tabular}{|c|c|c|c|c|c|c|c|}
\hline \multirow{2}{*}{ Pesticida } & \multicolumn{6}{|c|}{ Critérios EPA } & \multirow{2}{*}{ GUS } \\
\hline & Solubil. & $\mathrm{K}_{\mathrm{oc}}$ & $\mathrm{K}_{\mathrm{H}}$ & $\mathrm{t}_{1 / 2 \text { solo }}$ & $\mathrm{t}_{1 / 2 \text { água }}$ & Resultado & \\
\hline $2,4-\mathrm{D}$ & A & A & $\mathrm{A}$ & $\mathrm{N}$ & $\mathrm{N}$ & $\mathrm{PC}$ & $2,2 \mathrm{TL}$ \\
\hline Atrazina & A & A & $\mathrm{A}$ & A & $\mathrm{N}$ & $\mathrm{PC}$ & 3,2 PL \\
\hline Bispiribaque sódico & A & & A & $\mathrm{N}$ & A & $\mathrm{PC}$ & Zero IL \\
\hline Carbofurano & A & A & $\mathrm{A}$ & A & A & $\mathrm{PC}$ & 4,73 PL \\
\hline Carboxina & A & A & A & $\mathrm{N}$ & $\mathrm{N}$ & $\mathrm{PC}$ & Zero IL \\
\hline Ciclossulfamurom & $\mathrm{N}$ & $\mathrm{N}$ & & & $\mathrm{N}$ & $\mathrm{NC}$ & \\
\hline Clomazona & A & A & $\mathrm{A}$ & A & $\mathrm{N}$ & $\mathrm{PC}$ & 3,9 PL \\
\hline Cialofope-butilico & $\mathrm{N}$ & & $\mathrm{A}$ & $\mathrm{N}$ & & I & \\
\hline Fenoxaprope-p-etílico & $\mathrm{N}$ & & $\mathrm{A}$ & $\mathrm{N}$ & A & I & \\
\hline Fipronil & $\mathrm{N}$ & $\mathrm{N}$ & $\mathrm{A}$ & $\mathrm{N}$ & $\mathrm{N}$ & $\mathrm{NC}$ & $-0,4 \mathrm{IL}$ \\
\hline Glifosato & A & $\mathrm{N}$ & $\mathrm{A}$ & A & $\mathrm{N}$ & $\mathrm{PC}$ & $-0,6 \mathrm{IL}$ \\
\hline Lambda-cialotrina & $\mathrm{N}$ & $\mathrm{N}$ & $\mathrm{N}$ & A & $\mathrm{N}$ & $\mathrm{NC}$ & $-2,4 \mathrm{IL}$ \\
\hline Linurom & A & A & $\mathrm{A}$ & A & A & $\mathrm{PC}$ & 3,01 PL \\
\hline Metsulfurom-metílico & A & A & $\mathrm{A}$ & $\mathrm{A}$ & & $\mathrm{PC}$ & 4,2 PL \\
\hline Molinato & A & A & $\mathrm{N}$ & A & & $\mathrm{PC}$ & 3,8 PL \\
\hline Parationa-metílica & $\mathrm{A}$ & A & $\mathrm{N}$ & $\mathrm{A}$ & & $\mathrm{PC}$ & $2,1 \mathrm{TL}$ \\
\hline Pendimetalina & $\mathrm{N}$ & A & $\mathrm{N}$ & A & $\mathrm{N}$ & $\mathrm{NC}$ & 3,1 PL \\
\hline Permetrina & $\mathrm{N}$ & $\mathrm{N}$ & & A & $\mathrm{N}$ & $\mathrm{NC}$ & $-1,4 \mathrm{IL}$ \\
\hline Pirazossulfurom-etílico & $\mathrm{N}$ & & & A & $\mathrm{N}$ & $\mathrm{NC}$ & \\
\hline Propanil & A & A & $\mathrm{A}$ & $\mathrm{N}$ & $\mathrm{N}$ & $\mathrm{PC}$ & Zero IL \\
\hline Propinebe & $\mathrm{N}$ & & $\mathrm{A}$ & $\mathrm{N}$ & & $\mathrm{NC}$ & \\
\hline Quincloraque & $\mathrm{N}$ & A & $\mathrm{A}$ & & & I & \\
\hline Sulfosato & A & & & & & I & \\
\hline Tebuconazol & A & & $\mathrm{A}$ & & $\mathrm{N}$ & $\mathrm{PC}$ & \\
\hline Tiobencarbe & & $\mathrm{N}$ & $\mathrm{A}$ & A & & I & $1,4 \mathrm{IL}$ \\
\hline Tiram & $\mathrm{N}$ & A & $\mathrm{N}$ & A & $\mathrm{N}$ & $\mathrm{NC}$ & 4,7 PL \\
\hline Trifluralina & $\mathrm{N}$ & $\mathrm{N}$ & $\mathrm{N}$ & $\mathrm{A}$ & & $\mathrm{NC}$ & $0,75 \mathrm{TL}$ \\
\hline
\end{tabular}

EPA ( $\mathrm{N}=$ não atende ao critério; $\mathrm{A}=$ atende ao critério com potencial perigoso; $\mathrm{I}=$ inconclusivo; $\mathrm{PC}=$ contaminante em potencial; $\mathrm{NC}=$ não contaminante; em branco = dado não disponível; solubil. = solubilidade em água); GUS (IL = Não sofre Lixiviação; TL = Faixa de Transição; $\mathrm{PL}=$ Provável Lixiviação).

Ao investigar somente a água subterrânea, os pesticidas pendimetalina, tiram, 2,4-D, bisbiribaque sódico, carboxina, parationametílica, propanil e tebuconazol devem ser avaliados, pois são contaminantes em potencial destas águas (PC) e provavelmente lixiviados (PL), segundo os critérios da EPA e GUS respectivamente.

Portanto, estudos de monitoramento na região deverão, preferencialmente, enfocar a investigação nestes compostos, visto que são bastante utilizados na região e apresentam risco de contaminação aos corpos hídricos.

\section{MATERIAL SUPLEMENTAR}

No material suplementar disponível em http://quimicanova.sbq.org. br, na forma de arquivo PDF, com acesso livre e gratuito, encontram-se as seguintes tabelas: Tabela $1 \mathrm{~S}$ - Pesticidas utilizados nos municípios de Rio Grande e Santa Vitória do Palmar, com suas respectivas classes, fórmulas estruturais, nomes comerciais. Tabela $2 \mathrm{~S}$ - Principais propriedades físico-químicas dos pesticidas em estudo.

\section{AGRADECIMENTOS}

Ao CNPq, FAPERGS pelo auxílio financeiro. À $\operatorname{Prof}^{\mathrm{a}} \operatorname{Dr}^{\mathrm{a}} \mathrm{R}$. M. Clementin, pela revisão das estruturas dos pesticidas.

\section{REFERÊNCIAS}

1. Silva, C. M. M. S.; Fay, E. F.; Agrotóxicos Aspectos Gerais: Agrotóxicos e Ambiente, Embrapa: Brasília, 2004.

2. Caldas, E. D.; Souza, L. C. K.; Rev. Saúde Pública 2000, 34, 529.

3. Sanches, S. M.; Silva, C. H. T. P.; Campos, S. X.; Vieira, E. M.; Pesticidas: R. Ecotox. e Meio Ambiente 2003, 13, 53.

4. Veiga, M. M.; Silva, D. M.; Veiga, L. B. E.; Farias, M. V. C.; Cad. de Saúde Publica 2006, 22, 2391.

5. Ribeiro, M. L.; Lourencetti, C.; Pereira, S. Y.; Marchi, M. R. R.; Quim. Nova 2007, 30, 688.

6. Barceló, D.; Hennion, M. C.; Trace Determination of Pesticides and their degradation products in water, $2^{\text {nd }}$ ed., Elsevier: Amsterdam, 2003.

7. Tomita, R. Y.; Beyruth, Z.; Biologico 2002, 64, 135.

8. Carvalho, A. R.; Schlitter, F. H. M.; Tornisielo, V. L.; Quim. Nova 2000, 23,618 .

9. Armas, E. D.; Monteiro, R. T. R.; Quim. Nova 2005, 28, 975.

10. Lourencetti, C.; Spadotto, C. A.; Santiago-Silva, M.; Ribeiro, M. L.; Pesticidas: R. Ecotox. e Meio Ambiente 2005, 15, 1

11. Paraiba, L. C.; Cerdeira, A. L.; da Silva, E. F.; Martin, J. S.; Coutinho, H. L. C.; Chemosphere 2003, 53, 1087.

12. Worrall, F.; Kolpin, D. W.; J. Hydrology 2004, 293, 191. 
13. Coutinho, C. F. B.; Tanimoto, S. T.; Galli, A.; Gaberll, R. G. S.; Takayama, M.; Amaral, R. B.; Mazo, L. H.; Avaca, L. A.; Machado, S. A. S.; Pesticidas: R. Ecotox. e Meio Ambiente 2005, 15, 65.

14. http:/www.sindag.br/ acessada em Agosto 2007.

15. http:/www.irga.rs.gov.br/ acessada em Janeiro 2007.

16. http:/www.ibge.gov.br/ acessada em Fevereiro 2007.

17. Santos, I. R.; Monografia de Conclusão de Curso, Fundação Universidade Federal do Rio Grande, Brasil. 2003; Machado, G.; Rev. Discente Expressões Geográficas 2007, 3, 61.

18. http:/www.meteorologia.furg.br/acessada em Dezembro 2006.

19. Streck, E. V.; Kampf, N.; Dalmolin, R. S. D; Klamt, E.; Nascimento, P. C.; Scheneider, P.; Solos do Rio Grande do Sul, UFRS: Porto Alegre, 2002; http:/www.scp.rs.gov.br/atlas/ acessada em Dezembro 2007.

20. Dores, E. F. G. C., De-Lamonica-Freire, E. M.; Quim. Nova 2001, 24, 27.

21. Santos, T. C. R.; Rocha, J. C.; Barceló, D.; J. Chromatogr., A. 2000, 879, 3; Parreira F. V.; Paniago, E. B.; Carvalho C. R.; Afonso, R. J. C. F.; Pesticidas: R. Ecotox. e Meio Ambiente 2001, 11, 77; Laganà, A.; Bacaloni, A.; De Leva, I.; Fabri, A.; Fago, G.; Marino, A.; Anal. Chim. Acta 2002, 462, 187; Primel, E. G.; Zanella, R., Kurz, M. H. S.; Gonçalves, F. F.; Quim. Nova 2005, 28, 605; Marchesan, E.; Zanella, R.; Ávila, L. A.; Camargo, E. R.; Machado, S. L. O.; Macedo, V. R. M.; Sci. Agric. 2007, 64, 131.
22. Cerejeira, M. J.; Viana, P.; Batista, S.; Pereira, T.; Silva, E.; Valério, M. J.; Silva, A.; Ferreira, M.; Silva-Fernandes, A. M.; Water Res. 2003, 37, 1055; Guzzella L.; Pozzoni, F.; Guiliano, G.; Environ. Pollut. 2006, 142, 344; Raposo Jr., J. L.; Ré-Poppi, N.; Talanta 2007, 72, 1833.

23. Zanella, R. ; Grutzmacher, D. D.; Grutzmacher, A. D. ; Agostinetto, D. ; Loeck, A. E. ; Roman, R.; Resumo do $1^{\circ}$ Workshop Latino Americano de Resíduos de Pesticidas: Alimentos e Meio Ambiente, Santa Maria, Brasil, 2007

24. Fillmann, G.; Hermanns, L.; Fileman, T. W.; Readman, J. W.; Environ. Pollut. 2007, 146, 262.

25. Gonçalves, F.F.; Dissertação de Mestrado, Universidade Federal de Santa Maria, Brasil, 2007; Ávila, L. A.; Marchesan, E.; Zanella, R.; Camargo, E. R.; Machado, S. L. O.; Macedo, V. R. M.; Sci. Agric. 2007, 64, 137; Kurz, M. H. S.; Tese de Doutorado, Universidade Federal de Santa Maria, Brasil, 2007.

26. Scribner, E. A.; Bataglin W. A.; Goolsby, D. A.; Thurman, E. M.; Sci. Total Environ. 2000, 248, 255; Veiga M. M.; Silva, D. M.; Veiga, L. B. E.; Faria, M. V. C.; Cad. Saúde Pública 2006, 22, 2391; Shomar, B. H.; Muller, G.; Yahya, A.; Water, Air, Soil Pollut. 2006, 171, 237.

27. Albanis, T. A.; Konstantinou, I. H.; Sakkas, V. A.; Appl. Catal. B: Environ. 2001, 34, 227; Simal-Gándara, J.; Arias-Estévez, M.; LópezPeriago, E.; Martinez-Carballo, E.; Mejuto, J. C.; Garcia-Rio, L.; Agric. Ecosyst. Environ. 2008, 123, 247. 
ESTIMATIVA DE RISCO DE CONTAMINAÇÃO DAS ÁGUAS POR PESTICIDAS NA REGIÃO SUL DO ESTADO DO RS

Liziara Cabrera*, Fabiane Pinho Costa e Ednei Gilberto Primel

Escola de Química e Alimentos, Universidade Federal do Rio Grande, CP 475, 96201-900 Rio Grande - RS, Brasil

Tabela 1S. Pesticidas freqüentemente utilizados nos municípios de Rio Grande e Santa Vitória do Palmar

\begin{tabular}{|c|c|c|c|}
\hline $\begin{array}{l}\text { Princípio ativo } \\
\text { Classe }^{1}\end{array}$ & Estrutura $\mathbf{1 , 2}$ & Nomes comerciais ${ }^{3}$ & Grupo Químico 2,3 \\
\hline $\begin{array}{l}\# * 2,4-\mathrm{D} \\
\text { Herbicida }\end{array}$ & $\mathrm{Cl}$ & $\begin{array}{l}\text { Aminamar } \\
\text { Aminol } \\
\text { Capri }\end{array}$ & ácido ariloxialcanóico \\
\hline $\begin{array}{l}\text { \# Atrazina } \\
\text { Herbicida }\end{array}$ & & $\begin{array}{l}\text { Atranex } \\
\text { Coyote }\end{array}$ & triazina \\
\hline $\begin{array}{l}\text { *Bispiribaque sódico } \\
\text { Herbicida }\end{array}$ & & Nominee & ácido pirimidiniloxibenzóico \\
\hline $\begin{array}{l}\text { * Carbofurano } \\
\text { Acaricida, Inseticida, } \\
\text { Nematicida }\end{array}$ & & $\begin{array}{c}\text { Carboran Fersol } \\
\text { Diafuran } \\
\text { Furacarb } \\
\text { Ralzer }\end{array}$ & metilcarbamato de benzofuranila \\
\hline $\begin{array}{l}\text { \#Carboxina } \\
\text { Fungicida }\end{array}$ & & Vitavax & carboxanilida \\
\hline \#* Cialofope-butílico & & Clincher & ácido ariloxifenoxinroniônico \\
\hline $\begin{array}{l}\text { \# Ciclossulfamurom } \\
\text { Herbicida }\end{array}$ & & Invest & sulfoniluréia \\
\hline $\begin{array}{l}* \text { Clomazona } \\
\text { Herbicida }\end{array}$ & & $\begin{array}{c}\text { Gamit } \\
\text { Commence }\end{array}$ & isoxazolidinona \\
\hline $\begin{array}{l}\text { \#* Fenoxaprope- } \\
\text { p-etílico } \\
\text { Herbicida }\end{array}$ & & $\begin{array}{l}\text { Podium EW } \\
\text { Rapsode } \\
\text { Starice } \\
\text { Whip S }\end{array}$ & ácido ariloxifenoxipropiônico \\
\hline
\end{tabular}

*e-mail: liziara.cabrera@ vetorial.net 
Tabela 1S. continuação

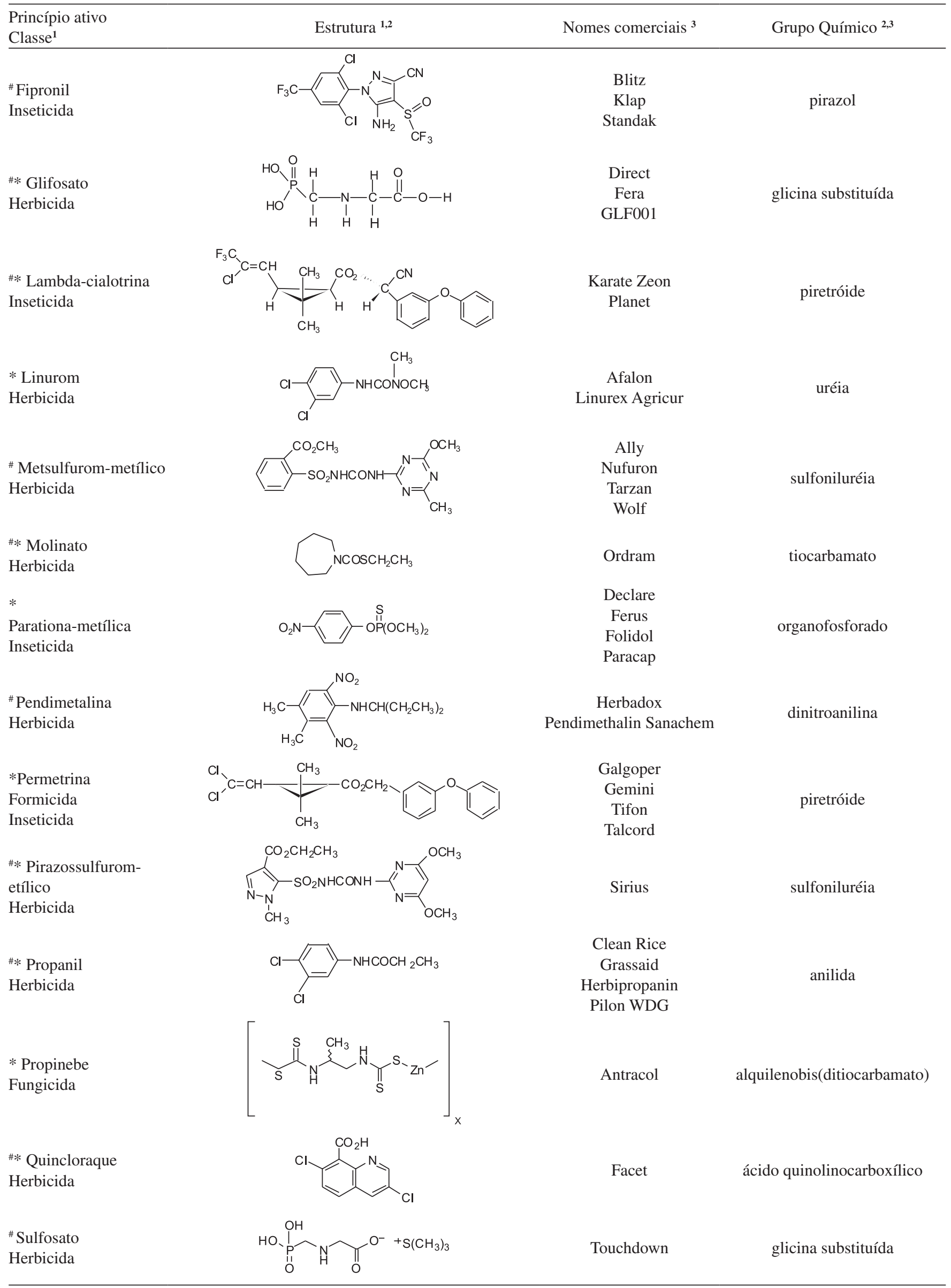


Tabela 1S. continuação

Princípio ativo
Classe ${ }^{1}$

* Rio Grande, " Sta Vitória do Palmar. 1. Tomlin, C.D.S., The e-Pesticide Manual, 13 ${ }^{\text {th }}$ ed. Versão 3.0. Londres. 2003-2004. 2. http:/www. anvisa.gov.br/AGROSIA/asp/frm_dados_ingrediente.asp/acessado em Janeiro 2007. 3. http:/www.agrofit/ acessada em Dezembro 2007.

Tabela 2 S. Propriedades físico-químicas dos pesticidas em estudo

\begin{tabular}{|c|c|c|c|c|c|c|c|c|}
\hline Pesticida & $\begin{array}{l}\text { Solubilidade em } \\
\text { água }\left(\mathrm{mg} \mathrm{L}^{-1}\right)^{1,2}\end{array}$ & $\begin{array}{c}\mathrm{K}_{\mathrm{oc}} \\
\left(\mathrm{cm}^{3} \mathrm{~g}^{-1}\right)^{1-7}\end{array}$ & $\log \mathrm{K}_{\mathrm{ow}}{ }_{1,2}$ & $\begin{array}{c}\text { PV } \\
(\mathrm{mPa})^{1,2}\end{array}$ & $\mathrm{pK}_{\mathrm{a}}^{1,2}$ & $\begin{array}{c}\mathrm{K}_{\mathrm{H}} \\
\left(\mathrm{Pam}^{3} \mathrm{~mol}^{-1}\right)^{1,2}\end{array}$ & $\begin{array}{l}\mathrm{t}_{1 / 2} \text { solo } \\
\text { (dias) }^{\mathbf{1 , 2 , 8}}\end{array}$ & $\begin{array}{l}\mathrm{t}_{1 / 2} \text { água } \\
\text { (dias) }^{\mathbf{1 , 2}}\end{array}$ \\
\hline $2,4-\mathrm{D}$ & $\begin{array}{l}20031(\mathrm{pH} \mathrm{5}), \\
34196(\mathrm{pH} 9)\end{array}$ & 39 & $2,6-2,8$ & $\begin{array}{c}1,9.10^{-2} \\
11\end{array}$ & 2,7 & $\begin{array}{c}1.10^{-5} \\
0,5\end{array}$ & 8 & 7,5 \\
\hline Atrazina & $33(\mathrm{pH} 7)$ & 24 & 2,5 & $3,8 \cdot 10^{-2}$ & 1,7 & $\begin{array}{c}1,5.10^{-4} \\
3.10^{-4}\end{array}$ & 50 & 55 \\
\hline Bispiribaque sódico & 73300 & & 1,0 & $5,0.10^{-6}$ & 3,0 & $3.10^{-11}$ & $<10$ & $\begin{array}{c}>365 \\
(\mathrm{pH} 7-9) \\
19 \\
(\mathrm{pH} 4) \\
>360 \\
(\mathrm{pH} 4)\end{array}$ \\
\hline Carbofurano & 320 & 22 & 1,52 & $0,031-0,072$ & & $5.10^{-4}$ & $\begin{array}{c}30-60 \\
50\end{array}$ & $\begin{array}{c}121 \\
(\mathrm{pH} 7) \\
1 \\
(\mathrm{pH} \mathrm{9})\end{array}$ \\
\hline Carboxina & 147 & 71 & 2,3 & $2,0.10^{-2}$ & $<0,5$ & $3.10^{-5}$ & $<1$ & $\begin{array}{c}0,6 \\
(\mathrm{pH} 7)\end{array}$ \\
\hline Cialofope-butilico & 0,5 (pH 5-7) & & 3,3 & $5,3 \cdot 10^{-2}$ & & $9.10^{-4}$ & $0,08-0,4$ & \\
\hline Ciclossulfamurom & $\begin{array}{l}0,2(\mathrm{pH} 5) \\
6,5(\mathrm{pH} 7) \\
549(\mathrm{pH} 9)\end{array}$ & 1440 & $\begin{array}{l}2,0(\mathrm{pH} 5) \\
1,4(\mathrm{pH} 7)\end{array}$ & $2.10^{-2}$ & 5,0 & & & $\begin{array}{c}0,3 \\
(\mathrm{pH} \mathrm{5)} \\
2 \\
(\mathrm{pH} 7-9)\end{array}$ \\
\hline Clomazona & 1100 & $150-560$ & 2,5 & 19 & & $4.10^{-3}$ & $30-135$ & $>30$ \\
\hline Fenoxaprope-p-etílico & $0,7(\mathrm{pH} \mathrm{6})$ & 9490 & $\begin{array}{c}0,8-0,2(\mathrm{pH} \\
5-9)\end{array}$ & $5.10^{-4}$ & & $3.10^{-4}$ & $1-10$ & $\begin{array}{c}>1000(\mathrm{pH} \\
5), \\
100 \\
(\mathrm{pH} 7)\end{array}$ \\
\hline
\end{tabular}


Tabela 2S. continuação

\begin{tabular}{|c|c|c|c|c|c|c|c|c|}
\hline Pesticida & $\begin{array}{l}\text { Solubilidade em } \\
\text { água }\left(\mathrm{mg} \mathrm{L}^{-1}\right)^{1,2}\end{array}$ & $\begin{array}{c}\mathrm{K}_{\mathrm{oc}} \\
\left(\mathrm{cm}^{3} \mathrm{~g}^{-1}\right)^{1-7}\end{array}$ & $\log \mathrm{K}_{\mathrm{ow}}{ }^{1,2}$ & $\begin{array}{c}\mathrm{PV} \\
(\mathrm{mPa})^{1,2}\end{array}$ & $\mathrm{pK}_{\mathrm{a}}^{1,2}$ & $\begin{array}{c}\mathrm{K}_{\mathrm{H}} \\
\left(\mathrm{Pam}^{3} \mathrm{~mol}^{-1}\right)^{1,2}\end{array}$ & $\begin{array}{l}\mathrm{t}_{1 / 2} \text { solo } \\
(\text { dias })^{\mathbf{1 , 2 , 8}}\end{array}$ & $\begin{array}{l}\mathrm{t}_{1 / 2} \text { água } \\
\text { (dias) }\end{array}$ \\
\hline Fipronil & $2(\mathrm{pH} 5-9)$ & 1248 & 4,0 & $4.10^{-4}$ & & $4.10^{-5}$ & $45-530$ & $\begin{array}{c}28 \\
(\mathrm{pH} 9)\end{array}$ \\
\hline Glifosato & $10500(\mathrm{pH} \mathrm{2})$ & 24.000 & & $1.10^{-2}$ & $2-10$ & $<2.10^{-7}$ & 47 & 91 \\
\hline $\begin{array}{l}\text { Lambda-cialotrina } \\
\text { (cialotrin) }\end{array}$ & $\begin{array}{l}5,0.10^{-3} \\
4,0.10^{-6}\end{array}$ & $3.10^{5}$ & 7,0 & $2.10^{-4}$ & $>9$ & $2.10^{-2}$ & $6-40$ & Rápido \\
\hline Linurom & $\begin{array}{c}63,8(\mathrm{pH} \mathrm{7}) \\
0,081\end{array}$ & $\begin{array}{c}500-600 \\
396\end{array}$ & $2,76-3$ & $\begin{array}{l}5.10^{-2} \\
5.10^{-5}\end{array}$ & & $\begin{array}{l}2.10^{-4} \\
5.10^{-3}\end{array}$ & $\begin{array}{c}38-67 \\
139\end{array}$ & 945 \\
\hline Metsulfurom-metílico & $\begin{array}{c}548(\mathrm{pH} \mathrm{5}) \\
2790(\mathrm{pH} \mathrm{7})\end{array}$ & 35 & $\begin{array}{c}0,02(\mathrm{pH} 7) \\
-1,7\end{array}$ & $3.10^{-7}$ & $\begin{array}{l}4 \\
3\end{array}$ & $\begin{array}{l}4.10^{-11} \\
(\mathrm{pH} 7)\end{array}$ & 52 & \\
\hline Molinato & $\begin{array}{c}990(\mathrm{pH} \mathrm{5}) \\
88\end{array}$ & 190 & 2,9 & 746 & & 0,1 & $40-160$ & \\
\hline Parationa-metílica & 55 & 236 & $\begin{array}{c}3,0 \\
1,8-3,04\end{array}$ & $2.10^{-6}$ & & $2.10^{-2}$ & 18,5 & \\
\hline Pendimetalina & 0,3 & 311 & 5,2 & $\begin{array}{c}2 \\
4.10^{-3}\end{array}$ & & 4 & $90-120$ & $<21$ \\
\hline Permetrina & $\begin{array}{c}6.10^{-3}(\mathrm{pH} 7) \\
0,2\end{array}$ & 10000 & $\begin{array}{c}6,1 \\
5,8-6,6\end{array}$ & $\begin{array}{c}\operatorname{cis} 2,5 \cdot 10^{-3} \\
\operatorname{trans} 1,5 \cdot 10^{-3} \\
4,5 \cdot 10^{-5}\end{array}$ & & & $\begin{array}{c}<38 \\
(\mathrm{pH} 4-8)\end{array}$ & $\begin{array}{c}50 \\
(\mathrm{pH} 9) \\
\text { estável (pH } \\
5-7)\end{array}$ \\
\hline Pirazossulfurom-etílico & 9,8 & & 3,2 & $4.10^{-5}$ & 3,7 & & $<15$ & 28 \\
\hline Propanil & 130 & $239-800$ & 3 & $\begin{array}{c}2.10^{-2}-5.10^{-2} \\
3.10^{-5}\end{array}$ & & $\begin{array}{l}2.10^{-4} \\
4.10^{-3}\end{array}$ & 1 & 0,5 \\
\hline Propinebe & 10 & & 0,26 & $<2.10^{-7}$ & & $8.10^{-8}$ & rápido & \\
\hline Quincloraque & $7.10^{-2}(\mathrm{pH} \mathrm{7})$ & 36 & $1(\mathrm{pH} 7)$ & $<1.10^{-2}$ & 4,3 & $4,4 \cdot 10^{-10}$ & & \\
\hline Sulfosato & 10000 & & $-2,9$ & $<1.10^{-2}$ & & & & \\
\hline Tebuconazol & 36 (pH 5-9) & & 3,7 & $2 \times 10^{-3}$ & & $1.10^{-5}$ & & $\begin{array}{c}7-28 \\
(\mathrm{pH} 4-9)\end{array}$ \\
\hline Tiobencarbe & 30 & 900 & 3,4 & $\begin{array}{c}3 \\
2200\end{array}$ & & & 21 & \\
\hline Tiram & 18 & 3 & 1,7 & 2,3 & & $3.10^{-2}$ & 23 & $\begin{array}{c}128 \\
(\mathrm{pH} 4)\end{array}$ \\
\hline Trifluralina & $0,184(\mathrm{pH} \mathrm{5)}$ & $4400-40000$ & 4,83 & 6,1 & & 15 & $57-126$ & \\
\hline
\end{tabular}

